PROCEEDINGS OF THE

AMERICAN MATHEMATICAL SOCIETY

Volume 139, Number 9, September 2011, Pages 3231-3245

S 0002-9939(2011)11031-1

Article electronically published on April 6, 2011

\title{
AREA EXTREMAL PROBLEMS FOR NON-VANISHING FUNCTIONS
}

\author{
T. SHEIL-SMALL
}

(Communicated by Mario Bonk)

\begin{abstract}
We consider the problem of finding the extremal function $f$ which minimises the Bergman space $A^{2}$ norm for the class of non-vanishing functions whose first $n+1$ Taylor coefficients are given. We define an analytic function $K$ in terms of $f$ and show that the functions $K$ and $f$ satisfy a certain differential equation. This equation yields a set of relationships between the area moments and the circle moments of $|f|^{2}$, which in particular shows that the outer part of $f$ is a polynomial of degree at most $n$.
\end{abstract}

\section{INTRODUCTION}

We consider the following class of problems. Let $f(z)=\sum_{k=0}^{\infty} a_{k} z^{k}$ be a function analytic in $U=\{|z|<1\}$ and satisfying $f(z) \neq 0$ in $U$ and $f(0)=a_{0}=1$. We wish to minimise the expression

$$
\int_{U}|f(z)|^{2} d A=\sum_{k=0}^{\infty} \frac{\left|a_{k}\right|^{2}}{k+1}
$$

under the hypotheses

$$
a_{k}=\alpha_{k} \text { for } 1 \leq k \leq n,
$$

where $\alpha_{k}$ are specified complex numbers and $d A=\frac{r}{\pi} d r d \theta$ is a normalised element of area. The more general problem, where, instead of specifying Taylor coefficients at the origin, we define $\alpha_{k}=l_{k}(f)$ as $n$ linearly independent continuous linear functionals, is considered in [1] and they study extensively the case when the linear functionals are evaluation functionals, i.e. what is given are relations of the form $f\left(\beta_{j}\right)=c_{j}(1 \leq j \leq n)$, the $\beta_{j}$ being points of $U$ and the $c_{j}$ arbitrary non-vanishing complex numbers. For the general case they show that an extremal function exists provided that the class is non-empty. That there are functions satisfying our conditions will be shown in section 6 . Thus our problem has at least one extremal function $f$ minimising the area integral. That this function is unique is shown by considering the existence of a second extremal function $g$ and considering the function $h=\sqrt{f g}$. It is easily shown that this function satisfies the conditions and using the uniqueness argument given in [1 we obtain $f=g$. We also require the general result of Aharonov and Shapiro that the extremal function is bounded in $U$. We will show that certain relationships are satisfied by the extremal function $f$. It will

Received by the editors March 14, 2010 and, in revised form, July 16, 2010.

2010 Mathematics Subject Classification. Primary 32A36; Secondary 30J99.

(C)2011 American Mathematical Society Reverts to public domain 28 years from publication 
be shown in a forthcoming paper that in the case $n=1$ these relationships enable us to determine the extremal function and thence find the extremal value.

\section{VARIATIONAL RESUlts}

Firstly for an arbitrary bounded analytic function $g(z)$ we consider the variation of $f$ given by

$$
\left(1+\epsilon g(z) z^{m}\right) f(z)
$$

where $m>n$ and $\epsilon$ is a complex constant sufficiently small so that $1+\epsilon g(z) z^{m} \neq 0$ in $U$. This function is a competing function; i.e. it is a non-vanishing function whose first $n+1$ coefficients are the same as those of $f$. From the minimum property of $f$ it follows that

$$
\int_{U}\left|1+\epsilon g(z) \mathrm{z}^{m}\right|^{2}|f(z)|^{2} d A \geq \int_{U}|f(z)|^{2} d A
$$

and hence

$$
2 \Re\left(\epsilon \int_{U} g(z) z^{m}|f(z)|^{2} d A\right)+|\epsilon|^{2} \int_{U}\left|z^{m} g(z)\right||f(z)|^{2} d A \geq 0 .
$$

Dividing by $|\epsilon|$ and letting $\epsilon \rightarrow 0$ we deduce that for any complex $\alpha$ satisfying $|\alpha|=1$ we have

$$
2 \Re\left(\alpha \int_{U} z^{m} g(z)|f(z)|^{2} d A\right) \geq 0
$$

and therefore

$$
\int_{U} z^{m} g(z)|f(z)|^{2} d A=0
$$

It is convenient here to define some notation. Let $\alpha=\left(\alpha_{0}, \alpha_{1}, \ldots, \alpha_{n}\right)$, where $\alpha_{0}=$ 1 , and define

$$
T(\alpha)=\int_{U}|f(z)|^{2} d A
$$

for the extremal function $f$ minimising this integral for functions whose first $n+1$ coefficients are $1, \alpha_{1}, \ldots, \alpha_{n}$. We also define

$$
m_{k}=\int_{U} z^{k}|f(z)|^{2} d A, \quad c_{k}=\frac{1}{2 \pi} \int_{0}^{2 \pi} e^{i k \theta}\left|f\left(e^{i \theta}\right)\right|^{2} d \theta
$$

for $k=0,1, \ldots$. Putting $g(z)=1$ we have from the above that

$$
m_{k}=0 \quad(k \geq n+1) .
$$

Theorem 2.1. We have the relationships

$$
m_{k}=\sum_{j=k}^{n} \bar{\alpha}_{j-k} \frac{\partial T}{\partial \bar{\alpha}_{j}}\left(\alpha_{0}, \ldots, \alpha_{n}\right) \quad(1 \leq k \leq n)
$$

holding whenever all the partial derivatives exist.

Proof. Here we are considering how the integrals $T(\alpha)$ vary as $\alpha$ varies. We are using the complex notation for partial differentiation so that if $\alpha_{j}=\beta_{j}+i \gamma_{j}$, then

$$
\frac{\partial}{\partial \bar{\alpha}_{j}}=\frac{1}{2}\left(\frac{\partial}{\partial \beta_{j}}+i \frac{\partial}{\partial \gamma_{j}}\right)
$$


We fix $k(1 \leq k \leq n)$ and consider the variation $\left(1+\epsilon z^{k}\right) f(z)$ for small complex $\epsilon$. This is a non-vanishing function which has the initial expansion

$$
\left(1+\epsilon z^{k}\right) f(z)=\sum_{j=0}^{k-1} \alpha_{j} z^{j}+\sum_{j=k}^{n}\left(\alpha_{j}+\epsilon \alpha_{j-k}\right) z^{j}+\ldots
$$

It follows that

$$
\int_{U}\left|1+\epsilon z^{k}\right|^{2}|f(z)|^{2} d A \geq T\left(\alpha_{0}, \ldots, \alpha_{k-1}, \alpha_{k}+\epsilon \alpha_{0}, \ldots, \alpha_{n}+\epsilon \alpha_{n-k}\right) .
$$

Hence

$$
\begin{aligned}
& 2 \Re\left(\int_{U} \epsilon z^{k}|f(z)|^{2} d A\right)+|\epsilon|^{2} \int_{U}\left|z^{k} f(z)\right|^{2} d A \\
& \quad \geq T\left(\alpha_{0}, \ldots, \alpha_{k-1}, \alpha_{k}+\epsilon \alpha_{0}, \ldots, \alpha_{n}+\epsilon \alpha_{n-k}\right)-T\left(\alpha_{0}, \ldots, \alpha_{n}\right) .
\end{aligned}
$$

Dividing by $|\epsilon|$ and letting $\epsilon \rightarrow 0$ we obtain the stated result as follows. We first take $\epsilon>0$ and then, assuming the existence of the partial derivatives, let $\epsilon \rightarrow 0$ obtaining an inequality with the real part of $m_{k}$. Next we take $\epsilon<0$ and letting $\epsilon \rightarrow 0$ this reverses the inequality. Thirdly we take $\epsilon$ purely imaginary with positive imaginary part and proceeding as before we obtain an inequality with the imaginary part of $m_{k}$. Finally we take $\epsilon$ imaginary with negative imaginary part reversing this inequality. The stated result now follows.

\section{The orthogonality Relationships}

Putting $g(z)=1$ in (3) we obtain

$$
\int_{U} z^{m}|f(z)|^{2} d A=0
$$

for $m \geq n+1$ and our extremal function $f$. We make use of the formula

$$
\int_{U} p(z) \overline{q(z)} d A=\frac{1}{2 \pi} \int_{0}^{2 \pi} p\left(e^{i \theta}\right) \overline{Q\left(e^{i \theta}\right)} d \theta,
$$

where if $q(z)=\sum_{k=0}^{\infty} q_{k} z^{k}$, then $Q(z)=\sum_{k=0}^{\infty} \frac{q_{k}}{k+1} z^{k}$, so $z Q^{\prime}+Q=q$. Both integrals exist if, for example, $p$ and $q$ are in $H^{2}$. For our case we have

$$
\int_{U} z^{m}|f(z)|^{2} d A=\frac{1}{2 \pi} \int_{0}^{2 \pi} e^{i m \theta} f\left(e^{i \theta}\right) \overline{F\left(e^{i \theta}\right)} d \theta=0
$$

for $m>n$, where $F(z)+z F^{\prime}(z)=f(z)$. This is equivalent to stating that the negative Fourier coefficients of the function $e^{i n \theta} f\left(e^{i \theta}\right) \overline{F\left(e^{i \theta}\right)}$ are 0 and therefore the harmonic extension into $U$ of this function is an analytic function in $U$. We can thus define an analytic function $K(z)$ by the formula

$$
\begin{array}{r}
K(z)=\frac{1}{2 \pi} \int_{0}^{2 \pi} \Re\left(\frac{1+z e^{-i t}}{1-z e^{-i t}}\right) e^{i n t} f\left(e^{i t}\right) \overline{F\left(e^{i t}\right)} d t \\
=\frac{1}{2 \pi} \int_{0}^{2 \pi} \frac{1}{1-z e^{-i t}} e^{i n t} f\left(e^{i t}\right) \overline{F\left(e^{i t}\right)} d t .
\end{array}
$$




\section{HARMONIC PRODUCTS AND THE MULTIPLICATION PROBLEM}

In order to make use of this result for $K(z)$ we need a concept of harmonic multiplication introduced in [3, chapter 4] whose main properties we outline below. If $p(z)=\sum_{k=-\infty}^{+\infty} p_{k} \sigma_{k}$ and $q(z)=\sum_{k=-\infty}^{+\infty} q_{k} \sigma_{k}$ are functions harmonic in $U$, where $\sigma_{k}=z^{k}$ for $k \geq 0$ and $=\bar{z}^{-k}$ for $k<0$, we define the harmonic product $p \bullet q$ by

$$
(p \bullet q)(z)=\sum_{j=-\infty}^{+\infty} \sum_{k=-\infty}^{+\infty} p_{j} q_{k} \sigma_{j+k}
$$

whenever this double series is absolutely convergent in $U$. Note that

(i) we always have $p \bullet q=q \bullet p$;

(ii) if $p$ and $q$ are analytic, then $p \bullet q$ exists and $p \bullet q=p q$;

(iii) $(p \bullet q)(z)=\sum_{i=-\infty}^{+\infty} r_{i} \sigma_{i}$ where

$$
r_{i}=\sum_{k=-\infty}^{+\infty} p_{i-k} q_{k}
$$

(iv) if $p$ and $q$ are in $h^{2}$, then $p \bullet q$ exists and

$$
|p \bullet q(z)| \leq\|p\|_{2}\|q\|_{2} \frac{1+|z|}{1-|z|} ;
$$

(v) if $p$ and $q$ are harmonic in $U$ and $0<\rho<1$, then $p(\rho z) \bullet q$ exists and

$$
(p(\rho z) \bullet q)=\lim _{R \rightarrow 1} \frac{1}{2 \pi} \int_{0}^{2 \pi} \Re\left(\frac{1+z e^{-i t}}{1-z e^{-i t}}\right) p\left(\rho e^{i t}\right) q\left(R e^{i t}\right) d t
$$

the limit existing locally uniformly in $U$ (see 3 for a proof of this); furthermore, if $q$ and $r$ are harmonic in $U$ and $q \bullet r$ exists, then

$$
(p(\rho z) \bullet q) \bullet r=p(\rho z) \bullet(q \bullet r) ;
$$

(vi) if $p$ and $q$ are in $h^{2}$, then

$$
p \bullet q(z)=\frac{1}{2 \pi} \int_{0}^{2 \pi} \Re\left(\frac{1+z e^{-i t}}{1-z e^{-i t}}\right) p\left(e^{i t}\right) q\left(e^{i t}\right) d t ;
$$

(vii) if $p$ and $q$ are in $h^{\infty}$ with $|p| \leq A$ and $|q| \leq B$, then $p \bullet q \in h^{\infty}$ and $|p \bullet q| \leq A B$. It follows that the binary operation $p \bullet q$ defines a complex linear algebra on $h^{\infty}$ and in particular

$$
(p \bullet q) \bullet r=p \bullet(q \bullet r)
$$

for $p, q$, and $r$ in $h^{\infty}$.

We now note that

$$
K(z)=z^{n} f(z) \bullet \overline{F(z)} .
$$

Lemma 4.1. Suppose $s \in H^{2}, r^{\prime} \in H^{2}$. Then $s^{\prime} \bullet \bar{r}$ exists and

$$
\left|\left(s^{\prime} \bullet \bar{r}\right)(z)\right| \leq \frac{2}{(1-|z|)^{2}}\|s\|_{2}\|\rho\|_{2},
$$

where $\rho=(z r)^{\prime}$. 
Proof. We have

$$
s^{\prime} \bullet \bar{r}=\sum_{j=1}^{\infty} \sum_{k=0}^{\infty} j s_{j} \overline{r_{k}} \sigma_{j-k-1}=\sum_{j=1}^{\infty} \sum_{k=0}^{\infty} j s_{j} \frac{\overline{\rho_{k}}}{k+1} \sigma_{j-k-1},
$$

where $\rho(z) \in H^{2}$. We obtain the estimate

$$
\begin{aligned}
\left|s^{\prime} \bullet r\right| & \leq \sum_{l} \sum_{k=0}^{\infty} \frac{k+1+l}{k+1}\left|s_{k+1+l} \| \rho_{k}\right||z|^{|l|} \\
& \leq \sum_{l}(|l|+1)|z|^{|l|}\|s\|_{2}\|\rho\|_{2} \leq \frac{2}{(1-|z|)^{2}}\|s\|_{2}\|\rho\|_{2},
\end{aligned}
$$

and thus the series for $s^{\prime} \bullet \bar{r}$ is absolutely and locally uniformly convergent in $U$.

Lemma 4.2. If $p \in H^{2}$ and $q \in H^{\infty}$, then

$$
p^{\prime} q=s^{\prime},
$$

where $s \in H^{2}$ and $\|s\|_{2} \leq\|q\|_{\infty}\|p\|_{2}$. On the other hand, if $p \in H^{\infty}$ and $q \in$ $H^{2}$, then

$$
p^{\prime} q=s^{\prime},
$$

where $s \in H^{2}$ and $\|s\|_{2} \leq 2\|q\|_{2}\|p\|_{\infty}$.

Proof. If $|q| \leq B$, we have $\left|s^{\prime}\right| \leq B\left|p^{\prime}\right|$ and hence

$$
\sum_{k=1}^{\infty} k^{2}\left|s_{k}\right|^{2} r^{2 k-2} \leq B^{2} \sum_{k=1}^{\infty} k^{2}\left|p_{k}\right|^{2} r^{2 k-2}
$$

for $0<r<1$. Replacing $r^{2}$ by $r$ and integrating we obtain

$$
\sum_{k=1}^{\infty} k\left|s_{k}\right|^{2} r^{k} \leq B^{2} \sum_{k=1}^{\infty} k\left|p_{k}\right|^{2} r^{k}
$$

Dividing by $r$ and integrating we obtain

$$
\sum_{k=1}^{\infty}\left|s_{k}\right|^{2} \leq B^{2} \sum_{k=1}^{\infty}\left|p_{k}\right|^{2}
$$

Defining $s(0)=0$ we obtain the first part of the result. For the second part we apply the first part to obtain $p q^{\prime}=t^{\prime}$, where $t \in H^{2}$ and $\|t\|_{2} \leq\|p\|_{\infty}\|q\|_{2}$. We also have

$$
p^{\prime} q=(p q)^{\prime}-p q^{\prime}=s^{\prime},
$$

where $s=p q-t$. Hence

$$
\|s\|_{2} \leq\|p q\|_{2}+\|t\|_{2} \leq 2\|p\|_{\infty}\|q\|_{2},
$$

as required.

From Lemmas 4.1 and 4.2 we obtain

Lemma 4.3. Let $r^{\prime} \in H^{2}$ and suppose either (a) $p \in H^{2}$ and $q \in H^{\infty}$ or (b) $p \in H^{\infty}$ and $q \in H^{2}$. Then $p^{\prime} q \bullet \bar{r}$ exists and

$$
\left|\left(p^{\prime} q \bullet \bar{r}\right)(z)\right| \leq \frac{2}{(1-|z|)^{2}}\|p\|_{2}\|q\|_{\infty}\|\rho\|_{2}
$$


in case (a) and

$$
\left|\left(p^{\prime} q \bullet \bar{r}\right)(z)\right| \leq \frac{4}{(1-|z|)^{2}}\|p\|_{\infty}\|q\|_{2}\|\rho\|_{2}
$$

in case (b), where $\rho=(z r)^{\prime}$.

Lemma 4.4. Let $p \in H^{2}, q \in H^{\infty}$ and $r^{\prime} \in H^{2}$. Suppose that $q \bullet \bar{r}$ is analytic in $U$. Then

$$
p^{\prime} q \bullet \bar{r}=p^{\prime}(q \bullet \bar{r}) .
$$

Proof. We consider $p^{\prime}(R z) q(z) \bullet \overline{r(z)}$, where $0<R<1$. As all three functions are bounded, we have

$$
p^{\prime}(R z) q(z) \bullet \overline{r(z)}=p^{\prime}(R z) \bullet(q \bullet \bar{r})=p^{\prime}(R z)(q \bullet \bar{r}),
$$

the last relation following from the hypothesis that $q \bullet \bar{r}$ is analytic. As $R \rightarrow 1$ the third expression converges locally uniformly in $U$ to $p^{\prime}(q \bullet \bar{r})$. Therefore to prove the lemma we need to show that

$$
p^{\prime}(R z) q(z) \bullet \overline{r(z)} \rightarrow p^{\prime} q \bullet \bar{r}
$$

locally uniformly in $U$. Equivalently

$$
\left(p^{\prime}(z)-R p^{\prime}(R z)\right) q(z) \bullet \overline{r(z)} \rightarrow 0
$$

as $R \rightarrow 1$, the convergence being locally uniform in $U$. But

$$
p^{\prime}(z)-R p^{\prime}(R z)=(p(z)-p(R z))^{\prime}
$$

and

$$
p(z)-p(R z)=\sum_{k=0}^{\infty}\left(1-R^{k}\right) p_{k} z^{k}
$$

and so by Lemma 4.3, case (a),

$$
\left|\left(p^{\prime}(z)-R p^{\prime}(R z)\right) q(z) \bullet \overline{r(z)}\right| \leq\|q\|_{\infty}\|p(z)-p(R z)\|_{2}\|\rho\|_{2} \frac{2}{(1-|z|)^{2}} .
$$

Thus to prove our lemma it remains to show that

$$
\sum_{k=0}^{\infty}\left(1-R^{k}\right)^{2}\left|p_{k}\right|^{2} \rightarrow 0 \text { as } R \rightarrow 1 .
$$

But

$$
\sum_{k=0}^{\infty}\left(1-R^{k}\right)^{2}\left|p_{k}\right|^{2} \leq\left(1-R^{N}\right)^{2} \sum_{k=0}^{N}\left|p_{k}\right|^{2}+\sum_{k=N+1}^{\infty}\left|p_{k}\right|^{2}
$$

and so we can choose $N$ so that the last sum is $<\epsilon$ and then note that for $R$ close to 1 the preceding expression is $<\epsilon$. This completes the proof.

Theorem 4.5. Let $f$ be our extremal function. Then

$$
\frac{f^{\prime}}{f}\left(z^{n} f \bullet \bar{F}\right)=z^{n} f^{\prime} \bullet \bar{F}
$$


Proof. Since $f$ is bounded and non-vanishing in $U$, we can write $f(z)=e^{-V(z)}$, where $V(0)=0$ and $\Re V(z)>-M$ in $U$ for some $M>0$. It follows that

$$
V(z)=\frac{2 M p(z)}{1-p(z)}
$$

where $p(0)=0$ and $|p(z)|<1$ in $U$. We obtain

$$
\frac{f^{\prime}(z)}{f(z)}=-V^{\prime}(z)=-\frac{2 M p^{\prime}(z)}{(1-p(z))^{2}}
$$

Applying Lemmas 4.3 and 4.4 with $q=z^{n} f$ and $r=F$ (possible since $(z F)^{\prime}=f$ and so $F^{\prime}$ is bounded) we deduce that $p^{\prime} q \bullet \bar{r}$ exists and

$$
p^{\prime} q \bullet \bar{r}=p^{\prime}(q \bullet \bar{r}) .
$$

This gives

$$
-(1-p(z))^{2} \frac{1}{2 M} \frac{f^{\prime}(z)}{f(z)}\left(z^{n} f \bullet \bar{F}\right)=-(1-p(z))^{2} \frac{1}{2 M} z^{n} f^{\prime} \bullet \bar{F} .
$$

Thus

$$
(1-p(z))^{2} \frac{f^{\prime}(z)}{f(z)}\left(z^{n} f \bullet \bar{F}\right)=(1-p(z))^{2} z^{n} f^{\prime} \bullet \bar{F} .
$$

Now $(1-p(z))^{2}$ is an outer function and therefore by Beurling's theorem is cyclic in $H^{2}$, which means that there exist polynomials $P_{m}$ such that

$$
\left\|P_{m}(z)(1-p(z))^{2}-1\right\|_{2} \rightarrow 0 \text { as } m \rightarrow \infty .
$$

Thus multiplying the last relation by $P_{m}$ we obtain

$$
\begin{gathered}
P_{m}(1-p(z))^{2} \frac{f^{\prime}(z)}{f(z)}\left(z^{n} f \bullet \bar{F}\right)=P_{m}\left((1-p(z))^{2} z^{n} f^{\prime} \bullet \bar{F}\right) \\
=P_{m} \bullet\left((1-p(z))^{2} z^{n} f^{\prime} \bullet \bar{F}\right)=\left(P_{m} \bullet(1-p(z))^{2} z^{n} f^{\prime}\right) \bullet \bar{F} \\
=P_{m}(1-p(z))^{2} z^{n} f^{\prime} \bullet \bar{F},
\end{gathered}
$$

where we have used the fact that $(1-p(z))^{2} z^{n} f^{\prime} \bullet \bar{F}$ is analytic and applied property (v). We have

$$
P_{m}(1-p(z))^{2} \frac{f^{\prime}(z)}{f(z)}\left(z^{n} f \bullet \bar{F}\right) \rightarrow \frac{f^{\prime}(z)}{f(z)}\left(z^{n} f \bullet \bar{F}\right) \text { as } m \rightarrow \infty
$$

locally uniformly in $U$. Thus to prove the theorem we need to show that

$$
P_{m}(1-p(z))^{2} z^{n} f^{\prime} \bullet \bar{F} \rightarrow z^{n} f^{\prime} \bullet \bar{F} \text { as } m \rightarrow \infty
$$

locally uniformly in $U$. From Lemma $4.3, z^{n} f^{\prime} \bullet \bar{F}$ exists, and so writing

$$
\epsilon_{m}=P_{m}(1-p(z))^{2}-1
$$

we need to show that

$$
\epsilon_{m} z^{n} f^{\prime} \bullet \bar{F} \rightarrow 0 \text { as } m \rightarrow \infty
$$

locally uniformly in $U$ under the hypothesis that

$$
\left\|\epsilon_{m}\right\|_{2} \rightarrow 0 \text { as } m \rightarrow \infty .
$$


From Lemma 4.3, case (b), we have

$$
\begin{aligned}
\left|\epsilon_{m} z^{n} f^{\prime} \bullet \bar{F}\right| & \leq\left\|\epsilon_{m}\right\|_{2}\|f\|_{\infty}\left\|(z F)^{\prime}\right\|_{2} \frac{4}{(1-|z|)^{2}} \\
& \rightarrow 0 \text { as } m \rightarrow \infty .
\end{aligned}
$$

This completes the proof of Theorem 4.5.

\section{THE DIFFERENTIAL EQUATION}

Theorem 5.1. Writing $K(z)=z^{n} f \bullet \bar{F}$ we have

$$
\begin{aligned}
z K^{\prime}(z)-\left(n+1+\frac{z f^{\prime}(z)}{f(z)}\right) K(z) & =-z^{n} f(z) \bullet \overline{f(z)} \\
& =-e^{\gamma} \phi(z) z^{n} \overline{\phi(1 / \bar{z})}
\end{aligned}
$$

where $\gamma>0$ is a constant and $\phi(z)$ is the outer part of $f$ and is a polynomial of degree at most $n$ with no zeros in $U$ and with $\phi(0)=1$.

To prove this we require the following differentiation formula.

Lemma 5.2. Suppose that $q \in H^{2}, r^{\prime} \in H^{2}$ and $q \bullet \bar{r}$ is analytic in $U$. Then

$$
(q \bullet \bar{r})^{\prime}=q^{\prime} \bullet \bar{r}-q \bullet \overline{z^{2} r^{\prime}} .
$$

Proof. We note that

$$
(q \bullet \bar{r})(z)=\sum_{j-k \geq 0} q_{j} \overline{r_{k}} z^{j-k},
$$

and therefore its derivative is given by

$$
(q \bullet \bar{r})^{\prime}(z)=\sum_{j-k \geq 1}(j-k) q_{j} \overline{r_{k}} z^{j-k-1} .
$$

The hypothesis is that

$$
\sum_{j-k<0} q_{j} \overline{r_{k}} z^{k-j}=0
$$

Now

$$
\left(q^{\prime} \bullet \bar{r}\right)(z)=\sum_{j, k} j q_{j} \overline{r_{k}} \sigma_{j-k-1},
$$

which exists by Lemma 4.1. Also

$$
\left(q \bullet \overline{z^{2} r^{\prime}}\right)(z)=\sum_{j, k} q_{j} k \overline{r_{k}} \sigma_{j-k-1},
$$

and therefore the difference between these quantities is

$$
\sum_{j, k}(j-k) q_{j} \overline{r_{k}} \sigma_{j-k-1} .
$$

It remains to show that

$$
\sum_{j-k<0}(j-k) q_{j} \overline{r_{k}} \bar{z}^{k-j-1}=0 .
$$

But this quantity is - (the derivative at $\bar{z}$ ) of

$$
\sum_{j-k<0} q_{j} \overline{r_{k}} z^{k-j}
$$

which is identically zero. Hence the lemma follows. 
To prove the theorem we use this formula on $K$, obtaining

$$
\begin{gathered}
K^{\prime}(z)=\left(z^{n} f\right)^{\prime} \bullet \bar{F}-z^{n} f \bullet \overline{z^{2} F^{\prime}} \\
=z^{n} f^{\prime} \bullet \bar{F}+n z^{n-1} f \bullet \bar{F}-z^{n-1} f \bullet \overline{z F^{\prime}} \\
=z^{n} f^{\prime} \bullet \bar{F}+(n+1) z^{n-1} f \bullet \bar{F}-z^{n-1} f \bullet \bar{f}
\end{gathered}
$$

since $F+z F^{\prime}=f$. Applying Theorem 4.5 gives us

$$
\begin{aligned}
z K^{\prime}(z) & =z\left(z^{n} f^{\prime} \bullet \bar{F}\right)+(n+1) K(z)-z^{n} f \bullet \bar{f} \\
& =\frac{z f^{\prime}}{f} K+(n+1) K-z^{n} f \bullet \bar{f},
\end{aligned}
$$

and we obtain the first equation of the above. In particular we deduce that $z^{n} f \bullet \bar{f}$ is analytic in $U$. Now, since $f(0)=1$, we write in slightly non-standard form,

$$
f(z)=\phi(z) S(z),
$$

where $\phi(z)$ is an outer function satisfying $\phi(0)=1$ and $S(z)$ is a singular function in the form

$$
S(z)=\exp \left(\gamma \frac{\omega(z)}{1+\omega(z)}\right)
$$

where $\gamma>0$ is a constant and where $\omega(z)$ is an inner function satisfying $\omega(0)=0$. In particular we have

$$
\omega(z) \overline{\omega(1 / \bar{z})}=1
$$

for all $z \in U$ and hence

$$
S(z) \overline{S(1 / \bar{z})}=e^{\gamma},
$$

from which we obtain

$$
S(z) \bullet \overline{S(z)}=e^{\gamma}
$$

We deduce that

$$
z^{n} f(z) \bullet \overline{f(z)}=e^{\gamma} z^{n} \phi(z) \bullet \overline{\phi(z)}
$$

Thus this function is analytic in $U$. Since $\phi$ is an outer function we can find a sequence of polynomials $P_{m}$ such that $\left\|P_{m} \phi-1\right\|_{2} \rightarrow 0$. So multiplying by $P_{m}$ and letting $m \rightarrow \infty$ we easily deduce that $z^{n} \bullet \overline{\phi(z)}$ is analytic in $U$. It follows immediately that $\phi$ is a polynomial of degree at most $n$, and hence we easily see that

$$
z^{n} \phi(z) \bullet \overline{\phi(z)}=\phi(z) z^{n} \overline{\phi(1 / \bar{z})} .
$$

This proves the theorem.

Note that the right-hand side of the differential equation is a polynomial of degree $2 n$.

Theorem 5.1. We have

$$
K(z)=z^{n} f(z) \overline{F(1 / \bar{z})}
$$

for all $z \in U$.

Proof. Firstly an inner function $I(z)$ satisfies $|I(z)|=1$ almost everywhere on the circle and therefore $I(z) \overline{I(1 / \bar{z})}=1$ almost everywhere on the circle. This formula may be used to extend $I(z)$ into the exterior of the disc so that the function becomes meromorphic in $\{|z|>1\}$ with poles at the conjugate points to the zeros of $I$ in $U$. Furthermore $I$ remains continuous on almost all rays from the origin. Now since $\phi(z)$ is an entire function and $S$ is singular, it follows that $f(z)$ can also be so 
continued and then becomes well-defined and analytic in $\{|z|>1\}$. By integration the same is true of $F(z)$. Define

$$
Q(z)=z^{n} f(z) \overline{F(1 / \bar{z})}
$$

so that by differentiation we obtain the differential equation

$$
\begin{gathered}
z Q^{\prime}(z)-\left(n+1+\frac{z f^{\prime}}{f}\right) Q(z)=-z^{n} f(z) \overline{f(1 / \bar{z})} \\
=-e^{\gamma} z^{n} \phi(z) \overline{\phi(1 / \bar{z})}
\end{gathered}
$$

and we obtain the same differential equation for $Q$ as for $K$. Thus writing $L=K-Q$ we deduce that

$$
z L^{\prime}(z)-\left(n+1+\frac{z f^{\prime}}{f}\right) L(z)=0 .
$$

Solving this gives us either $L \equiv 0$ or

$$
L(z)=e^{C} z^{n+1} f(z),
$$

where $C$ is a constant. But at almost all points on the unit circle, $Q=K$ and therefore $L=0$. Since $f$ cannot vanish almost everywhere on the circle, it follows that $L \equiv 0$ as asserted.

\section{The RELATionships}

Writing $\phi(z)=\prod_{k=1}^{n}\left(1+w_{k} z\right)$, we have

$$
z^{n} \phi(z) \overline{\phi(1 / \bar{z})}=\prod_{k=1}^{n}\left(1+w_{k} z\right)\left(\overline{w_{k}}+z\right)=\sum_{k=0}^{2 n} \lambda_{k} z^{k} .
$$

We also write

$$
K(z)=\sum_{k=0}^{\infty} d_{k} z^{k} \text { and } \frac{f^{\prime}(z)}{f(z)}=\sum_{k=0}^{\infty} b_{k} z^{k} .
$$

From the definition of $K$ we have

$$
d_{k}=\frac{1}{2 \pi} \int_{0}^{2 \pi} e^{i(n-k) \theta} f\left(e^{i \theta}\right) \overline{F\left(e^{i \theta}\right)} d \theta
$$

and observe that for $0 \leq k \leq n$ this gives

$$
d_{k}=\int_{U} z^{n-k}|f(z)|^{2} d A=m_{n-k} .
$$

We also have for $0 \leq k \leq n$,

$$
e^{\gamma} \lambda_{k}=\frac{1}{2 \pi} \int_{0}^{2 \pi} e^{i(n-k) \theta}\left|f\left(e^{i \theta}\right)\right|^{2} d \theta=c_{n-k} .
$$

The differential equation implies the following relationships obtained by equating coefficients. For $1 \leq k \leq 2 n$ we have

$$
(k-n-1) d_{k}-\sum_{j=0}^{k-1} b_{k-j-1} d_{j}=-c_{n-k}=-e^{\gamma} \lambda_{k},
$$

and for $k=0$ we get

$$
(n+1) d_{0}=(n+1) m_{n}=c_{n}=e^{\gamma} \lambda_{0} .
$$


In the case $k=n+1$ we obtain

$$
\sum_{j=0}^{n} b_{n-j} d_{j}=\overline{c_{1}}=e^{\gamma} \lambda_{n+1}
$$

Hence

$$
\sum_{j=0}^{n} b_{j} m_{j}=\overline{c_{1}}=e^{\gamma} \lambda_{n+1}
$$

For $0 \leq k \leq n-1$ we get

$$
(k+1) m_{k}+\sum_{j=k+1}^{n} b_{j-k-1} m_{j}=c_{k}=e^{\gamma} \lambda_{n-k} .
$$

We thus obtain $n+2$ relations between the $n+1$ quantities $m_{k}$, the $n+1$ quantities $c_{k}$ and the $n$ quantities $w_{k}$. Of course we also have the remaining relations for the coefficients $d_{k}$, but for $k>n$ it is not clear how to relate these to the $m_{k}$ in the area integrals. In the cases $k>2 n$ we obtain

$$
(n-k+1) d_{k}+\sum_{j=0}^{k-1} b_{k-j-1} d_{j}=c_{n-k}=\overline{c_{k-n}}=0 .
$$

We also observe that we need to know the coefficients $b_{k}$ of $f^{\prime} / f$ for $0 \leq k \leq n$. Writing $P(z)=1+\sum_{k=1}^{n} \alpha_{k} z^{k}$ we have

$$
f(z)=P(z)+z^{n+1} R(z),
$$

from which we obtain

$$
\frac{f^{\prime}(z)}{f(z)}=\frac{P^{\prime}(z)}{P(z)}+z^{n} S(z)
$$

which is valid near the origin since $P(0)=1$. It follows that the coefficients $b_{0}, \ldots, b_{n-1}$ depend only on the $\alpha_{k}(1 \leq k \leq n)$. Therefore these coefficients are fixed by the problem. A similar argument shows that $b_{n}$ is determined by the $\alpha_{k}$ and the coefficient $a_{n+1}$ of $f$. Only one of the relationships above between the $m_{k}$ and the $\lambda_{k}$ includes the term $b_{n}$ and therefore this is giving us unique information about the coefficient $a_{n+1}$. On the other hand, writing $f^{\prime}=f\left(f^{\prime} / f\right)$ we easily obtain the formula

$$
(k+1) a_{k+1}=\sum_{j=0}^{k} a_{k-j} b_{j}
$$

for $k=0,1, \ldots$. From this we see that given $b_{j}(0 \leq j \leq n-1)$ we can determine $a_{k}(1 \leq k \leq n)$ taking $a_{0}=1$. Thus it is a completely equivalent problem to assign the values $b_{k}(0 \leq k \leq n-1)$ instead of $a_{k}(1 \leq k \leq n)$. As a corollary of these remarks we can prove the existence of bounded non-vanishing functions $f$ whose first $n+1$ coefficients are the $\alpha_{k}$ with $\alpha_{0}=1$. We define the $b_{k}$ for $0 \leq k \leq n-1$ in terms of the $\alpha_{k}(1 \leq k \leq n)$ as above by expanding $P^{\prime}(z) / P(z)$ near the origin and then defining our function $f$ by

$$
f(z)=\exp \left(\sum_{k=0}^{n-1} \frac{b_{k}}{k+1} z^{k+1}\right) .
$$


Then $f$ is clearly non-vanishing in the plane and bounded in any disc. Furthermore

$$
\frac{f^{\prime}(z)}{f(z)}=\sum_{k=0}^{n-1} b_{k} z^{k}
$$

Thus by the construction of the $b_{k}$,

$$
f(z)=1+\sum_{k=1}^{n} \alpha_{k} z^{k}+\sum_{k=n+1}^{\infty} \tau_{k} z^{k}
$$

for certain coefficients $\tau_{k}$. Notice also that the argument given here shows that for the class of non-vanishing functions $\{g\}$ satisfying our $\alpha_{k}$ condition the class $\{\log g\}$ is a convex class. In particular, if $g$ and $h$ are two such functions, then $\sqrt{g h}$ is also such a function.

\section{The CONSTRUCtion OF A POTENTIAL EXTREMAL FUNCTION}

In this final section we attempt to construct a competing function $f$ which satisfies our differential equation and therefore acts as a potential extremal for the class. Our method is to consider an anti-derivative $G$ of $f$ and assume that this function has the same singular part as $f$. Thus $G=z F+C$ for a suitable constant $C$ and $G=\psi S$, where $\psi$ is bounded and has no singular part. Then

$$
f=G^{\prime}=\left(\psi^{\prime}+\psi \frac{S^{\prime}}{S}\right) S=\phi S .
$$

This gives

$$
\phi=\psi^{\prime}+\psi \frac{S^{\prime}}{S} .
$$

Now the function $K(z)$ defined as the harmonic extension into $U$ of $z^{n} f(z) \overline{F(z)}$ on $T$ needs to be analytic and so $z^{n+1} f(z) \overline{G(z)}$ has no co-analytic part on $T$. But this implies that $z^{n+1} \phi(z) \overline{\psi(z)}$ has no co-analytic part and therefore, since $\phi$ is an outer function and so cyclic, the function $z^{n+1} \overline{\psi(z)}$ has no co-analytic part on $T$. It follows that $\psi$ is a polynomial of degree at most $n+1$. Writing $S=e^{\gamma \omega /(1+\omega)}$, where $\gamma>0$ and $\omega$ is an inner function, we observe that $S^{\prime} / S$ is rational and therefore $\omega$ is a finite Blaschke product. It follows that

$$
\frac{\omega(z)}{1+\omega(z)}=\sum_{k=1}^{m} \lambda_{k} \frac{z e^{-i t_{k}}}{1+z e^{-i t_{k}}},
$$

where $\lambda_{k}>0$ and $\sum_{k=1}^{m} \lambda_{k}=1$. We therefore obtain

$$
\phi(z)=\psi^{\prime}(z)+\psi(z) \sum_{k=1}^{m} \gamma \lambda_{k} \frac{e^{-i t_{k}}}{\left(1+z e^{-i t_{k}}\right)^{2}} .
$$

It follows that

$$
\psi(z)=\sigma(z) \prod_{k=1}^{m}\left(1+z e^{-i t_{k}}\right)^{2},
$$

where $\sigma$ is a polynomial of degree at most $n+1-2 m$. In particular the degree of the Blaschke product $\omega$ is at most $\frac{1}{2}(n+1)$. Now the function $K(z)$ is determined 
by the formula

$$
K(z)+C z^{n+1} f(z)=\phi(z) z^{n+1} \overline{\psi(1 / \bar{z})}
$$

and we note that the right-hand side is a polynomial of degree at most $2 n+1$. From the construction this will satisfy our differential equation with the function $f=\phi S$ provided that $f \neq 0$ in $U$. In other words, we need to show that by a suitable choice of the parameters $\gamma, t_{k}, \lambda_{k}$ and the polynomial $\sigma$, the polynomial $\phi$ defined by the above equation is non-vanishing in $U$. Furthermore $f$ must have its initial coefficients as the $\alpha_{k}$. This is equivalent to assigning values to the coefficients $b_{k}$ of $f^{\prime} / f$ for $0 \leq k \leq n-1$. Now

$$
\frac{f^{\prime}}{f}=\frac{\phi^{\prime}}{\phi}+\sum_{j=1}^{m} \gamma \lambda_{j} \frac{c_{j}}{\left(1+c_{j} z\right)^{2}},
$$

where $c_{j}=e^{-i t_{j}}$, so if we write

$$
\phi(z)=\prod_{i=1}^{n}\left(1+w_{i} z\right)
$$

we have

$$
b_{k}=(-1)^{k} \sum_{j=1}^{n} w_{j}^{k+1}+\sum_{i=1}^{m} \gamma \lambda_{i}(k+1) c_{i}^{k+1} ;
$$

and so the question is, can we solve these equations for $\lambda_{i}, c_{i}$ and $w_{j}$, with $\left|w_{j}\right| \leq 1$, where $b_{k}$ are arbitrary given numbers and $\gamma \geq 0$ ?

We consider the simplest possible case; namely $\omega$ has degree 1 and so $m=1$. This is the case where $S$ has its measure $\mu$ with a single atom, and a result of Caughran [2] shows that in this case the anti-derivative $G+C$ for a suitable constant $C$ has the same singular part $S$. The polynomial $\sigma$ will have degree at most $n-1$ and satisfies the equation

$$
\phi(z)=c(2+\gamma+2 c z) \sigma(z)+(1+c z)^{2} \sigma^{\prime}(z) .
$$

If $\phi_{k}$ and $\sigma_{k}$ are the coefficients of $\phi$ and $\sigma$, we obtain the following relations:

$$
\phi_{k}=(k+1) c^{2} \sigma_{k-1}+c(2 k+2+\gamma) \sigma_{k}+(k+1) \sigma_{k+1}
$$

for $0 \leq k \leq n$, where we take $\sigma_{-1}=0$ and $\sigma_{k}=0$ for $k \geq n$. If we choose $\phi$ as a polynomial with no zeros in $U$, we can solve this equation for the $\sigma_{k}$ by starting with $k=n$ which defines $\sigma_{n-1}$ and proceeding backwards to $k=1$. For $k=0$ we have

$$
1=c(2+\gamma) \sigma_{0}+\sigma_{1},
$$

and providing that $\sigma_{0} \neq 0$ we obtain

$$
\frac{1-\sigma_{1}}{\sigma_{0}}=c(2+\gamma)
$$

This defines $c$ but also requires that

$$
\frac{\left|1-\sigma_{1}\right|}{\left|\sigma_{0}\right|} \geq 2
$$


in order that we obtain $\gamma \geq 0$. The case $\gamma=0$ means there is no singular part to $f$, and this occurs iff the polynomial

$$
P(z)=1+\sum_{k=1}^{n} \alpha_{k} z^{k}
$$

has no zeros in $U$. In this case $f(z)=P(z)$ is the extremal and we obtain $\phi(z)=$ $P(z)$ and $S(z)=1$. Now in order to define $\phi$ we need to solve the equations

$$
b_{k}=(-1)^{k} \sum_{j=1}^{n} w_{j}^{k+1}+\gamma(k+1) c^{k+1}
$$

for $0 \leq k \leq n-1$, where the $b_{k}$ are arbitrary but given complex numbers and the choices of $\gamma \geq 0$ and $c(|c|=1)$ are open. Having done this we obtain the polynomial $\sigma$ as above, but then find that $\gamma$ and $c$ are determined by (49) and a further constraint (50) between $\sigma_{0}$ and $\sigma_{1}$ must be satisfied. Thus it appears that many, but not all, cases can be covered with a single atom function. However, we make the tentative conjecture that for the extremal function $f$ the anti-derivative $G+C$ does have the same singular part as $f$ for a suitable constant $C$.

\section{Concluding Remarks}

The above conjecture essentially amounts to an assumption of "niceness" in one of the anti-derivatives $G=z F+C$. If it could be shown that the non-singular part of $G$ is analytic in the closed disc and the measure appearing in the singular part is purely atomic, then indeed $f$ and $G$ have the same singular part. However, there is (as yet) no a priori reason for believing this to be the case. However. it is the simplest assumption for actually constructing solutions to the differential equation and works beautifully in the case $n=1$ to produce a single prospective extremal function with the value of the integral easily calculated from the relationships. Nevertheless the proof that this is the actual extremal function makes very subtle use of the relationships, requires further use of the extremal nature of the function and finally uses the missing variation given in Theorem 2.1, which is the main reason we have included this rather complicated result.

The main result of this paper can be generalised to the cases where what is given are the values of a set of linear functionals each of which is a finite linear combination of Taylor coefficients evaluated at a finite number of specified points in the disc. Two conditions are required: (i) there is at least one function satisfying the conditions, not necessarily non-vanishing but which is non-vanishing at the specified points; (ii) the identically zero function does not satisfy all the conditions. Then at least one non-vanishing extremal function exists and all extremal functions are bounded and have rational outer part of a particular form. Furthermore we obtain a generalised differential equation. This work will appear in a forthcoming paper.

Finally, the work of this paper was greatly inspired by the work of Aharonov, Bénéteau, Khavinson and Shapiro [1. In addition to the mathematical work, there is a good amount of historical reference to the preceding literature on non-vanishing functions in Hardy spaces and to linear extremal problems in Bergman spaces. The reader may wish to consult this paper for a substantial list of references. 


\section{REFERENCES}

[1] Dov Aharonov, Catherine Bénéteau, Dmitry Khavinson and Harold Shapiro, Extremal problems for nonvanishing functions in Bergman spaces, Operator Theory: Advances and Applications, 158, Birkhäuser, 2005, 59-86. MR2147588 (2006i:30047)

[2] J. Caughran, Factorisation of analytic functions with $H^{p}$ derivatives, Duke Math. J. 36 (1969), 153-158. MR0239095 (39:454)

[3] T. Sheil-Small, Complex Polynomials, Cambridge Studies in Advanced Mathematics, 75, Cambridge University Press, 2002. MR1962935 (2004b:30001)

Department of Mathematics, University of York, Heslington, York, YO 10 5DD, UnITED KingDOM

Current address: P. O. Box 60681, CY8106, Paphos, Cyprus 